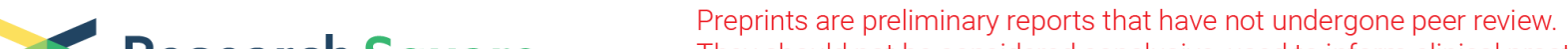 Research Square They should not be considered conclusive, used to inform clinical practice, or referenced by the media as validated information.
}

\section{Evaluation of the classification and clinical characteristics of oral lichen planus and its treatment effect in different clinical types: a retrospective study}

Atsushi Abe ( $\square$ atsushi.a@ekisai.or.jp )

Nagoya Ekisaikai Hospital

Yu Ito

Aichi Gakuin University

Hiroki Hayashi

Nagoya Ekisaikai Hospital

Moeko Momokita

Nagoya Ekisaikai Hospital

Shinichi Taniguchi

Nagoya Ekisaikai Hospital

Atsushi Nakayama

Aichi Gakuin University

\section{Research Article}

Keywords: oral lichen planus, clinical classification, oral potentially malignant disorders, oral

Posted Date: February 2nd, 2022

DOI: https://doi.org/10.21203/rs.3.rs-1314235/v1

License: (a) (i) This work is licensed under a Creative Commons Attribution 4.0 International License.

Read Full License 


\section{Abstract}

Background. Oral lichen planus (OLP) is an intractable chronic inflammatory disease associated with dyskeratosis. Several clinical classifications of OLP have been reported. However, only a few detailed studies have investigated the clinical symptoms, classifications, and treatment effect. In this study, we investigated the clinical features of OLP at the time of the initial diagnosis and classified it using the clinical visual examination type classification. Furthermore, we evaluated whether there is a difference in the clinical characteristics and effectiveness of symptomatic treatment between the typical reticular type and other types of OLP.

Methods. A total of 132 patients with OLP were included. Patients' age, sex, chief complaints, clinical morphological forms, lesion sites, cytological and histopathological findings, therapeutic strategies, and therapeutic responsiveness were evaluated.

Results. Of the 132 patients with OLP, there were 42 men and 90 women, with a mean age of $66.4 \pm 13.0$ years. The buccal mucosa was the most affected site $(n=95)$. The reticular form was the most common clinical morphological form, observed in 124 cases. Biopsy was performed in 48 patients. The erosive form was the most common among the biopsied lesions. Regarding therapeutic strategies, topical steroids were prescribed to 85 patients. Among these patients, 47 experienced symptomatic relief and 38 showed no changes in symptoms. Based on the clinical morphological form, the rates of symptomatic relief following topical steroid use were $70.4 \%$ for the reticular form and $15.9 \%$ for the erosive form. In patients with erosive and plaque-like forms, it was difficult to achieve symptomatic relief with topical steroid use, whereas in those with other forms, a moderate response was observed.

Conclusion: Topical steroids are often ineffective in patients with the erosive or mixed form, and these clinical morphological forms are associated with a higher risk of malignant transformation compared with the other forms. Therefore, early histological examination is important.

Trial registration number: Not applicable.

\section{Introduction}

Oral lichen planus (OLP) is a chronic intractable inflammatory disease associated with abnormal keratinization of the oral mucosa [1-3]. The age of onset varies worldwide, and a higher incidence has been observed in women. Furthermore, smokers and alcohol abusers have a higher prevalence of OLP. The cause remains unknown, although the immunological system plays a substantial role, and several factors are involved, including psychological stress and drug intake. OLP may also be associated with several systemic diseases, such as hepatitis C, diabetes mellitus, graft-versus-host disease, and thyroid dysfunction. The risk of malignancy is $1.2 \%$ [4]. The exact clinical features of the majority of OLPs remain unclear [5]. OLP presents with a variety of clinical features, including reticulation, erosions, papules, plaques, and bullae, and several classification methods have been reported [3-4, 6-7]. Although these classification methods can clearly classify typical cases, secondary infections and cases in which 
multiple types are involved may not be easily identified because of variability among diagnosticians [8]. Symptoms of OLP are variable, with approximately two-thirds of patients complaining of burning sensation and pain in the oral mucosa [9]. Among the different clinical forms, reticular OLP is the most common. The clinical manifestations of OLP differ greatly depending on the type, with the reticular type causing only a feeling of roughness in the mucous membranes and the erosive type resulting in severe pain. Other types are associated with redness and are difficult to distinguish from other oral diseases.

Symptomatic treatment is the mainstay of therapy and includes gargling with mouthwash and topical application of steroids. However, the treatment efficacy varies depending on the clinical type. In many cases, topical application is effective, although in some cases, there may be a relapse after a short period of remission.

In this study, we investigated the clinical features of OLP at the time of the initial diagnosis (clinical OLP) and classified it using the clinical visual examination type classification. Furthermore, we evaluated whether there is a difference in the clinical characteristics and effectiveness of symptomatic treatment between the typical reticular type and other types of OLP.

\section{Methods}

A total of 132 patients who visited the Department of Oral and Maxillofacial Surgery, Nagoya Subspecialty Hospital during the past 7 years from January 2013 to December 2019 and were diagnosed with OLP based on gross examination and clinical findings were included in this study.

The following variables were retrospectively examined using medical records for all cases: 1) patient age and sex, 2) chief complaint, 3) clinical visual examination type classification, 4) location of the lesion, 5) whether cytological and histopathological examinations were performed, and 6) treatment method and response to the treatment. Lesions with presumed or confirmed causes, such as allergy to dental materials or drugs or graft versus host disease, were diagnosed as lichen planus-like lesions and excluded from this study. The location of the lesions was classified as intrinsic buccal mucosa, alveolar gingiva, gingiva-buccal transition, buccal mucosa to gingival-buccal transition, tongue, palate, and lips. The most representative findings of the lesions were the clinico-epithelial type according to Andreasen's classification [4]. Histopathological examination was performed at the site of the most representative lesion as far as possible. Usually, the main treatment methods for OLP include the use of medicinal mouthwash and oral cleaning instructions, topical application of steroid ointment, oral cepharanthine, vitamin $\mathrm{A}$ and $\mathrm{B} 6$ administration, oral steroids, dental metal removal, and traditional Chinese medicine. This study evaluated the prescription of topical steroids in patients with OLP, and the response to the treatment was evaluated based on the perceived improvement and change in the lesion on visual examination.

This study was approved by the Ethics Committee of Nagoya Suburban Hospital (approval no. 2019057), and written informed consent was obtained from all participants. 


\section{Statistical analyses}

Continuous variables were evaluated for normality using the Shapiro-Wilk test. Normally distributed data are reported as means and standard deviations. Differences in means were evaluated using the paired $t$ test, and non-normally distributed data were analyzed using Wilcoxon's signed rank test. Categorical data were evaluated using the chi-square test, and general responses were reported descriptively. Statistical significance was set at $p<0.05$.

\section{Results}

\section{Age distribution by sex}

The mean age of the patients was $66.4 \pm 13.0$ years. The male to female ratio was $1: 2.1$, with $42(31.8 \%)$ men and $90(68.2 \%)$ women. Of the total patients, 46 (32.1\%) were in their $70 \mathrm{~s}, 30(23.2 \%)$ in their $60 \mathrm{~s}$, and $22(21.2 \%)$ in their 50 s, followed by those in their 40 s and 80 s (Figure 1 ).

\section{Reason for clinic visit and the chief complaint}

The most common reason for visiting the clinic was a request for a close examination of white or red lesions (69 cases, $52.2 \%$ ), followed by contact pain in 40 cases (30.2\%), burning sensation in $18(13.9 \%)$, and others (eating disorder due to pain and others) in 5 (3.7\%) (Figure 2).

\section{Clinically visual type of lesion}

According to Andreasen's classification [4], we classified the lesions into six clinical types: reticular, erosive, atrophic, plaque, papular, and bullous. Of the total, 124 patients $(43 \%)$ had reticular lesions, 95 $(33 \%)$ had erosive lesions, $5(2 \%)$ had plaque lesions, $5(2 \%)$ had papular lesions, $10(3 \%)$ had atrophic lesions, 3 (1\%) had bullous lesions, and 47 (16\%) had mixed lesions (Figure 3).

\section{Frequency of lesions by site}

The total number of lesion sites was 289, and the mean number of lesion sites per case was 2.19. The sites of occurrence were the intrinsic buccal mucosa in 95, the alveolar gingiva in 71, the gingival-buccal transition area in 47 , the tongue in 42 , the buccal mucosa-gingival-buccal transition area in 19 , the palate in 8 , and the lips in 7 . The number of lesions per site in the clinico-visual classification was examined. The reticular type was most common in the intrinsic buccal mucosa, alveolar gingiva, tongue, palate, and lips, followed by the erosive type. In contrast, the erosive and mixed types were more common than the reticular type in the buccal mucosa-gingival-buccal transition and gingival-buccal transition areas. Of the total patients, $77(58.3 \%)$ had bilateral lesions and $55(41.7 \%)$ had unilateral lesions (Figure 4). 


\section{Histopathological diagnosis}

Cytological diagnosis was performed in 91 of the 132 cases. The cytological diagnosis was negative in 81 cases and suspicious positive in 10.

Biopsy was performed in 48 of 132 cases (36.4\%) and was most commonly performed for erosive lesions. Twenty-eight cases (68.8\%) were histopathologically diagnosed as OLP because of characteristic histological changes, such as 1) liquefaction and degeneration of basal cells, 2) exfoliation from connective tissue, 3) and 4) colloid bodies. Of the 12 cases with pathological diagnoses other than OLP, 5 were diagnosed as keratosis, 4 as ulcers, 2 as squamous cell carcinomas, and 1 as pemphigus vulgaris (Figure 5 and Table 1).

Table 1

Number of cytological and histopathological examinations performed

$$
\text { Cytology }
$$

Performed Not performed

Suspected positive Negative

$\begin{array}{lll}10 & 81 & 45\end{array}$

Histopathological examination

performed Not performed

OLP OLP compatible No OLP

\section{Treatment methods}

$\begin{array}{llll}28 & 8 & 12 & 84\end{array}$

Eighty-five patients (64.4\%) were prescribed topical steroids, and 47 (35.6\%) were only prescribed thylaminium chloride mouthwash and oral cleaning instructions. Forty-seven of the 85 patients (55.3\%) showed improvement in symptoms with steroid ointment, and 38 (44.7\%) showed no change in symptoms. In addition, 19 (40.4\%) of the 47 cases showed improvement in symptoms after rinsing and oral hygiene instructions, and 28 (59.6\%) showed no change in symptoms. The improvement rate of symptoms with steroid ointment was 50 of 71 cases $(70.4 \%)$ in the reticular type, 7 of $44(15.9 \%)$ in the erosive type, 10 of $24(41.7 \%)$ in the plaque-like type, 10 of $16(62.5 \%)$ in the papular type, 10 of 14 $(71.4 \%)$ in the atrophic type (71.4\%), 2 of $2(100 \%)$ in the bullous type, and 8 of $14(57.1 \%)$ in the mixed type. Steroid ointment did not improve the symptoms in the erosive and plaque-type lesions but was somewhat effective in the other lesions. The symptom improvement rate without steroid ointment was 28 of $59(47.5 \%)$ cases in the reticular type, 2 of $5(40.0 \%)$ in the erosive type, 2 of $8(42.9 \%)$ in the plaquelike type, 6 of $14(42.9 \%)$ in the papular type, 2 of $10(20.0 \%)$ in the atrophic type, 0 of $2(0 \%)$ in the bullous type, and 2 of $6(0 \%)$ in the mixed type (Figure $6 a, b)$. 


\section{Discussion}

OLP is a chronic inflammatory disease with refractory dyskeratosis and is one of the most frequent oral mucosal diseases [10-13]. Although several studies have been conducted on OLP, the etiology is unknown and treatment can be difficult [1]. OLP is more common in middle-aged and older women in their 40 s to 60 s, with a male-to-female ratio of approximately $1: 2$ to $1: 3$. In this study, the ratio of males to females was 1:2.1, and more than half of the patients were in their $60 \mathrm{~s}$ or $70 \mathrm{~s}$, indicating that OLP was more prevalent in older individuals in this study. The reason for the high prevalence of OLP in women is unclear; however, some reports have shown little or no sexual predilection $[9,14]$. These results should be interpreted after considering the possibility that many male patients have latent OLP and do not have the opportunity to be examined because of regional bias or lack of time for hospital visits because of work. In addition, Japan has a large aging population and is expected to become a "super-aged" society in the future. With the extension of healthy life expectancy and longer life expectancy of women, the prolonged duration of the disease will pose a risk for carcinogenesis, which is a major issue.

The subjective symptoms of this lesion include mild pain, contact and irritation pain, burning sensation, and discomfort of the oral mucosa. In general, unpleasant symptoms, such as irritation, contact pain, and burning sensation, are observed in approximately half of the patients $[14,15]$. In addition, it has been reported that approximately $30 \%$ of the patients without clear symptoms of discomfort present to the hospitals because they are motivated by mass media reports or were aware of some abnormality, such as a feeling of coarseness [3]. Currently, the speed of information dissemination through the Internet and media is remarkably fast, and patients can easily obtain medical information, including correct and incorrect information. The differential diagnosis of this disease from cancer and precancerous lesions is important. From the viewpoint of preventive medicine, there is a need to further educate both medical professionals and the public. In this study, approximately half of the patients had no subjective symptoms and were examined only for the purpose of diagnosis as they visited our department for a thorough examination. More than half of the patients presented at an early disease stage, within one month of the onset of symptoms, while others visited more than one year after the onset of symptoms.

The most frequent site of OLP in the oral cavity is the cheek mucosa (approximately $35 \%$ ), followed by the gingiva and tongue, while the palate is relatively rarely affected $[2-4,6-7,15,16]$. Some reports have classified the location of the lesions into the buccal mucosa and gingiva in a broad sense, although they are often found mainly at the gingiva-buccal mucosa transition area. These lesions usually persist for a long period [3] and are affected by the state of the periodontal tissues. Therefore, in this study, the gingiva-buccal mucosa transition area was included in the classification according to the location. The results showed that the most common site was the buccal mucosa, followed by the alveolar gingiva, gingiva-buccal mucosa transition area, tongue, and intrinsic buccal mucosa to gingiva-buccal mucosa transition area. Bilateral lesions were found in $57.7 \%$ of cases, with a mean lesion size of $2.1 \mathrm{~cm}$, indicating multiple lesions, a trend consistent with those of previous reports $[3,8,15,16]$. The most common form of OLP is a typical lace-like white patch on the buccal mucosa with surrounding redness, which is relatively easy to distinguish. However, OLP lesions on the gingiva and tongue may be modified 
by secondary infections or irritation from teeth or prosthetic materials, making them difficult to differentiate.

There are several clinico-visual classifications of OLP lesions, including the Andreasen classification [4], Silverman's classification [7], and Einsen's classification [17]. It is important that the classification is consistent among the evaluators and is easy and reliable for the diagnosis of a wide range of mixed lesions. In this study, the Andreasen classification, which has several classification items and is widely used for external validity, was used [4]. In this study, as in other reports, the reticular type was the most common, followed by the erosive type [4]. Comparing the chief complaint with the type classification of clinical visual examination, the reticular type is generally less symptomatic than the other types. The atrophic, erythematous, erosive, and ulcerative types tend to be associated with more unpleasant symptoms, such as contact pain, than the reticular and hyperkeratotic types [16]. Several other studies have reported similar results, with cases involving ulcers and erosions being symptomatic [18-23]. However, the issues with the classification of clinical visual types vary according to the reports of each institution, there are no clear diagnostic criteria, and the reproducibility and agreement among institutions and diagnosticians are not always high. OLP, especially the reticular type, can be easily diagnosed by the naked eye. However, several other lesions, such as leukoplakia, erythroplakia, and epithelioid type, show findings similar to those of OLP, and unified diagnostic criteria have not been developed to distinguish these lesions. Histopathological examination is essential for the definitive diagnosis of OLP. However, many institutions diagnose clinical OLP based on gross examination and palpation and do not perform a biopsy. In a review of cases clinically diagnosed as OLP, biopsy was performed in $35.8 \%$ of cases, and it is thought that cases that are relatively easy to diagnose clinically, such as the reticular type, are less problematic. In a report on the concordance of pathological diagnosis with clinical diagnosis of OLP, the concordance rate was reported to be approximately $50-80 \%[7,17,24-27]$. In this study, cytological diagnosis was performed in 91 cases (73.4\%) and biopsy was performed in 48 (38.7\%); the percentage of cases where biopsy was performed was equivalent to that reported by other studies. It is important to detect malignancy early in the treatment and follow-up of this disease, and it is necessary for the surgeon to decide whether to use cytology for simplicity or biopsy for definitive diagnosis. In addition, clinical and pathological diagnoses are often inconsistent, and technical problems are encountered during biopsy, such as the inability to obtain a reliable pathological diagnosis because the biopsy was performed at a site with poor tissue response rather than at the site of the main lesion. OLP has been reported to undergo malignant transformation, and although the mechanism of malignant transformation is unknown, it has been suggested that increased inflammatory cytokines may be involved [12]. The clinical classification of malignant lesions has been reported to be erosive or erythematous [17]. Therefore, in cases of erosive or atrophic lesions, biopsy at the time of initial diagnosis and shorter follow-up intervals are necessary. In all cases of malignant transformation, clinical findings, such as enlargement of erythema and worsening of contact pain, were observed during the follow-up from the initial diagnosis to malignant transformation $[12,28-32]$. Biopsy should be performed when morphological and color changes or clinical symptoms appear during the follow-up. 
In our department, OLP is treated with oral hygiene instructions and follow-up, and local steroid therapy is initiated when clinical symptoms, such as spontaneous pain, appear. If a fungal infection is detected, antifungal drugs are administered, and steroid ointment is discontinued until no fungal infection is detected. In this study, the effectiveness of steroid ointment in improving symptoms in patients with OLP was evaluated. The use of steroid ointment was found to improve symptoms in all but erosive and papular lesions. It is desirable to evaluate symptom improvement using objective indices, such as a sign score [33] or visual analog scale (VAS). However, it has been reported that the size of the lesion does not correlate with the intensity of subjective symptoms and that the validity of VAS evaluation is questionable because of its large variability [3]. This study used subjective and visual changes of the lesion as criteria for judging improvement by treatment [3]. Furthermore, the improvement of the lesions based on the patient's awareness and change in the appearance of lesions on visual examination were evaluated. In a previous study on the treatment effect of the clinical examination type of the lesions, there was no difference in the treatment effect on reticular, erosive, and atrophic lesions, the treatment effect in mixed reticular-erosive lesions was poor, and the lesions were more resistant to treatment than other types [3]. In this study, steroid ointment did not improve symptoms in the erosive and plaque-shaped lesions but was somewhat successful in other types. This may have been because of the extent and size of the erosions and abrasion caused by the positional relationship between the teeth and prosthesis.

This study has some limitations. It involved a retrospective analysis of data from a single facility. Furthermore, the sample size used in this study was not sufficient. OLP may be associated with Candida infection, which may reduce the efficacy of topical steroid application; however, this was not investigated in this study. However, the number of cases of the atrophic, papular, plaque-like, and bullous forms of lichen planus evaluated in this study was small, and more cases should be included future studies.

\section{Conclusion}

In this study, we clinically evaluated 124 patients with OLP. Even when clinically diagnosed as OLP, the erosive form of OLP, especially when of the mixed type including the erosive form, often did not respond to steroid treatment and had a higher risk of malignant transformation than other clinically diagnosed types. Therefore, early histological examination is important.

\section{Abbreviations}

OLP, oral lichen planus

VAS, visual analog scale

\section{Declarations}

\section{Ethics approval and consent to participate}


All procedures were conducted in accordance with the ethical standards of the institutional and/or national research committees and in line with the 1964 Declaration of Helsinki. This retrospective cohort study was approved by the Institutional Review Board of Nagoya Suburban Hospital, and written informed consent was obtained from all participants (approval no. 2019-057). This study was conducted in accordance with the Strengthening the Reporting of Observational Studies in Epidemiology (STROBE) statement, a guideline for reporting observational studies. The Ethics Committee approved the procedures of this study and granted administrative permission to access the data used in this study.

\section{Consent for publication}

Not applicable.

\section{Availability of data and materials}

The raw data are confidential and cannot readily be shared. Researchers need to obtain permission from the Institutional Review Board and apply for access to the data from The Ethics Committee of Nagoya Ekisaikai Hospital.

\section{Competing interests}

The authors declare that they have no competing interests.

\section{Funding}

The present research did not receive any specific grant from funding agencies in the public, commercial, or not-for-profit sectors.

\section{Authors' contributions}

AA conceived the study, carried out the design and coordination, wrote the manuscript, and gave the final approval of the version to be submitted. YI and AN critically revised the manuscript for important intellectual content. HH, ST, and MM collected the clinical data and drafted the article. All authors read and approved the final manuscript.

\section{Acknowledgements}

We would like to thank Editage Science Communications for English language editing and publication support.

\section{References}

1. Scully C, Carrozzo M. Oral mucosal disease: lichen planus. Br J Oral Maxillofac Surg. 2008;46:1521. 
2. McCartan BE, Healy CM. The reported prevalence of oral lichen planus: a review and critique. J Oral Pathol Med. 2008;37:447-53.

3. Eisenberg E, Krutchkoff DJ. Lichenoid lesions of oral mucosa. Diagnostic criteria and their importance in the alleged relationship to oral cancer. Oral Surg Oral Med Oral Pathol. 1992;73 73:699-704.

4. Andreasen JO. Oral lichen planus. A clinical evaluation of 115 cases. Oral Surg Oral Med Oral Pathol. 1968;25:31-42.

5. 5.. Li C, Tang X, Zheng X, Ge S, Wen H, Lin X, et al. Global prevalence and incidence estimates of oral lichen planus: a systematic review and meta-analysis. JAMA Dermatol. 2020 Feb 1;156:172-81. doi: 10.1001/jamadermatol.2019.3797. PMID: 31895418; PMCID: PMC6990670.

6. Axéll T, Rundquist L. Oral lichen planus-a demographic study. Community Dent Oral Epidemiol. 1987;15:52-6.

7. Silverman S Jr, Gorsky M, Lozada-Nur F. A prospective follow-up study of 570 patients with oral lichen planus: persistence, remission, and malignant association. Oral Surg Oral Med Oral Pathol. 1985;60:30-4.

8. Boñar-Alvarez P, Pérez Sayáns M, Garcia-Garcia A, Chamorro-Petronacci C, Gándara-Vila P, LucesGonzález R, et al. Correlation between clinical and pathological features of oral lichen planus: a retrospective observational study. Med (Baltim). 2019;98:e14614. doi:10.1097/MD.0000000000014614.

9. Mehta FS, Gupta PC, Daftary DK, Pindborg JJ, Choksi SK. An epidemiologic study of oral cancer and precancerous conditions among 101,761 villagers in Maharashtra, India. Int J Cancer. 1972;10:13441.

10. Carrozzo M, Thorpe R. Oral lichen planus: a review. Minerva Stomatol. 2009;58:519-37.

11. Ismail SB, Kumar SKS, Zain RB. Oral lichen planus and lichenoid reactions: etiopathogenesis, diagnosis, management and malignant transformation. J Oral Sci. 2007;49:89-106.

12. van der Waal I. Oral lichen planus and oral lichenoid lesions; a critical appraisal with emphasis on the diagnostic aspects. Med Oral Patol Oral Cir Bucal. 2009;14:E310-4.

13. Shirasuna K. Oral lichen planus: malignant potential and diagnosis. Oral Sci Int. 2014;11:1-7.

14. Reichart PA. Oral mucosal lesions in a representative cross-sectional study of aging Germans. Community Dent Oral Epidemiol. 2000;28:390-8.

15. Scardina GA, Messina P. Morphological characteristics of microcirculation in oral lichen planus involving the lateral border of the tongue. J Oral Sci. 2009;51:193-7. doi:10.2334/josnusd.51.193.

16. Mankapure PK, Humbe JG, Mandale MS, Bhavthankar JD. Clinical profile of 108 cases of oral lichen planus. J Oral Sci. 2016;58:43-7. doi:10.2334/josnusd.58.43.

17. Eisen $D$. The clinical features, malignant potential, and systemic associations of oral lichen planus: a study of 723 patients. J Am Acad Dermatol. 2002;46:207-14. 
18. Jolehar M, Mohseni R, Farhadi S. Correlation between WHO and modified WHO classification systems in the histopathologic diagnosis of oral lichen planus using intraobserver and interobserver variability. Int J Prev Med. 2021 Sep 29;12:126. doi: 10.4103/ijpvm.IJPVM_566_18. PMID: 34760137; PMCID: PMC8551796.

19. Radochová V, Koberová Ivančaková R, Heneberk O, Slezák R. The characteristics of patients with oral lichen planus and malignant transformation-a retrospective study of 271 patients. Int $\mathrm{J}$ Environ Res Public Health. 2021 Jun 17;18:6525. doi: 10.3390/ijerph18126525. PMID: 34204361; PMCID: PMC8296493.

20. Elsabagh HH, Gaweesh YY, Ghonima JK, Gebril M. A novel comprehensive scoring system for oral lichen planus: a validity, diagnostic accuracy, and clinical sensitivity study. Oral Surg Oral Med Oral Pathol Oral Radiol. 2021 Mar;131:304-11. doi: 10.1016/j.00oo.2020.12.016. Epub 2020 Dec 20. PMID: 33451971.

21. Daume L, Kreis C, Bohner L, Kleinheinz J, Jung S. Does the clinical form of oral lichen planus (OLP) influence the oral health-related quality of life (OHRQoL)? Int J Environ Res Public Health. 2020 Sep 11;17:6633. doi: 10.3390/ijerph17186633. PMID: 32932968; PMCID: PMC7559187.

22. Feldmeyer L, Suter VG, Oeschger C, Cazzaniga S, Bornstein MM, Simon D, et al. Oral lichen planus and oral lichenoid lesions - an analysis of clinical and histopathological features. J Eur Acad Dermatol Venereol. 2020 Feb;34:e104-7. doi: 10.1111/jdv.15981. Epub 2019 Dec 15. PMID: 31568593.

23. Carrozzo M, Porter S, Mercadante V, Fedele S. Oral lichen planus: a disease or a spectrum of tissue reactions? Types, causes, diagnostic algorhythms, prognosis, management strategies. Periodontol 2000. 2019 Jun;80:105-25. doi: 10.1111/prd.12260. PMID: 31090143.

24. van der Meij EH, van der Waal I. Lack of clinicopathologic correlation in the diagnosis of oral lichen planus based on the presently available diagnostic criteria and suggestions for modifications. J Oral Pathol Med. 2003;32:507-12.

25. Onofre MA, Sposto MR, Navarro CM, Motta ME, Turatti E, Almeida RT. Potentially malignant epithelial oral lesions: discrepancies between clinical and histological diagnosis. Oral Dis. 1997;3:148-52.

26. van der Meij EH, Schepman KP, Plonait DR, Axéll T, van der Waal I. Interobserver and intraobserver variability in the clinical assessment of oral lichen planus. J Oral Pathol Med. 2002;31:95-8.

27. Brocklehurst P, Kujan O, O'Malley LA, Ogden G, Shepherd S, Glenny AM. Screening programmes for the early detection and prevention of oral cancer. Cochrane Database Syst Rev. 2013;11. doi:10.1002/14651858.CD004150.pub4..

28. Naggar El, AK, Reichart PA. Proliferative verrucous leukoplakia and precancerous conditions. In: Pathology and genetics head and neck tumours. Barnes, I, Eveson, JW, Reichart, P, editors. Lyon: IARC Press; 2005. p. 180-1.

29. Bombeccari GP, Guzzi G, Tettamanti M, Giannì AB, Baj A, Pallotti F, et al. Oral lichen planus and malignant transformation: a longitudinal cohort study. Oral Surg Oral Med Oral Pathol Oral Radiol Endod. 2011;112:328-34. 
30. Gonzalez-Moles MA, Scully C, Gil-Montoya JA. Oral lichen planus: controversies surrounding malignant transformation. Oral Dis. 2008;14:229-43.

31. Gandolfo S, Richiardi L, Carrozzo M, Broccoletti R, Carbone M, Pagano M, et al. Risk of oral squamous cell carcinoma in 402 patients with oral lichen planus: a follow-up study in an Italian population. Oral Oncol. 2004;40:77-83.

32. Schlosser BJ. Lichen planus and lichenoid reactions of the oral mucosa. Dermatol Ther. 2010;23:251-67.

33. Thongprasom K, Luangjarmekorn L, Sererat T, Taweesap W. Relative efficacy of fluocinolone acetonide compared with triamcinolone acetonide in treatment of oral lichen planus. J Oral Pathol Med. 1992;21:456-8.

\section{Figures}

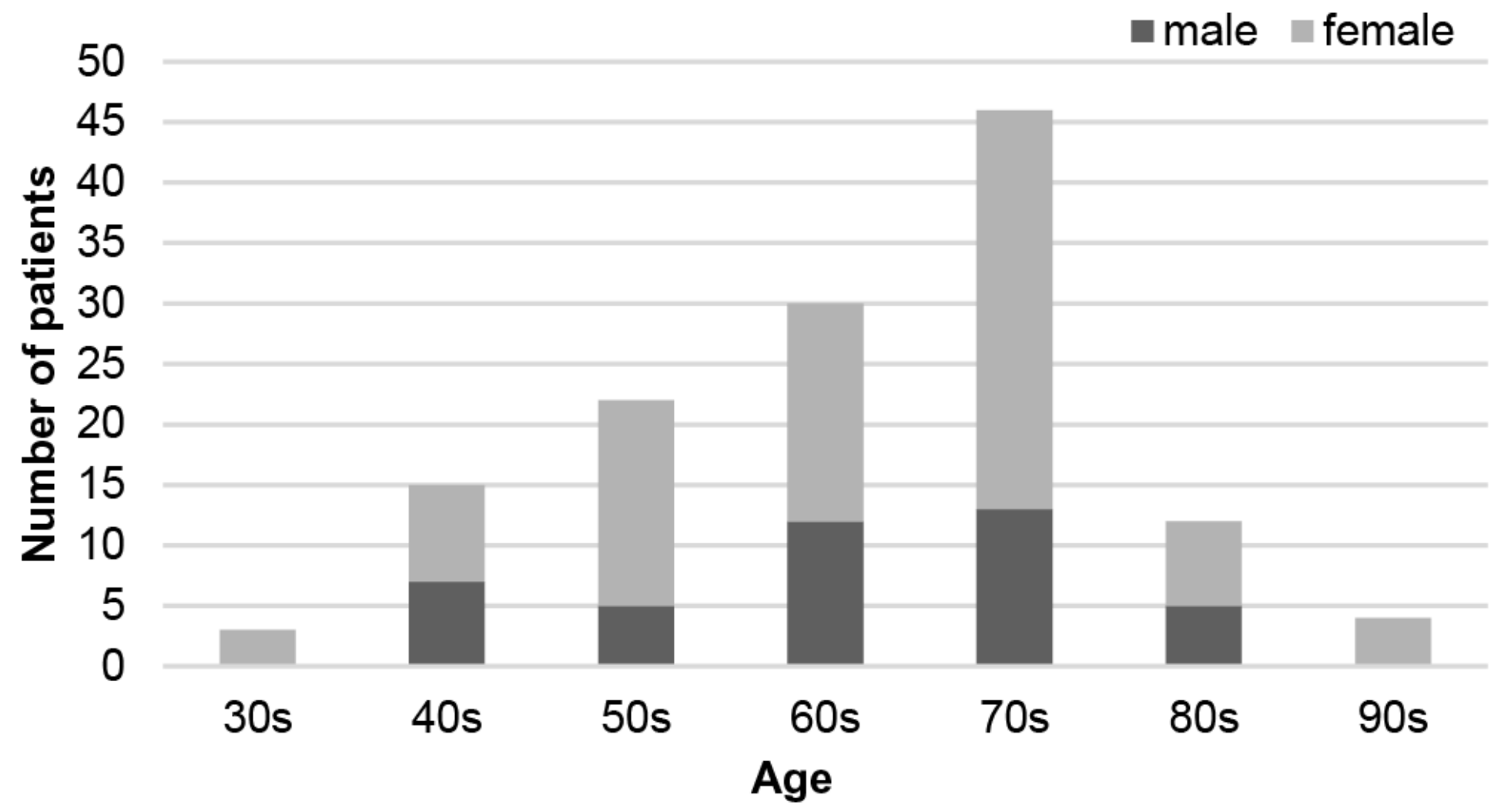

Figure 1

Age distribution of target patients 


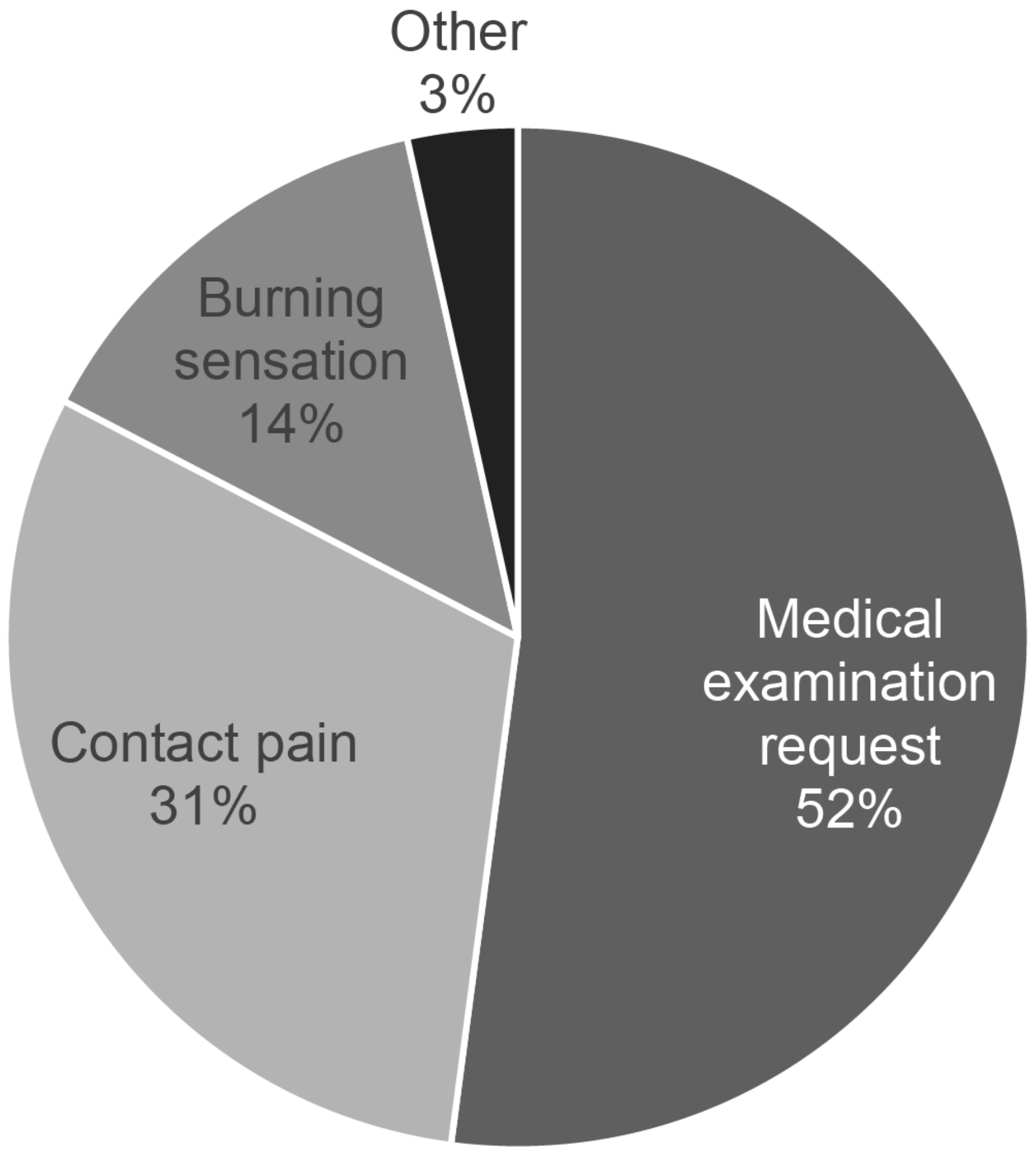

Figure 2

Reason for coming to the hospital and main complaint 


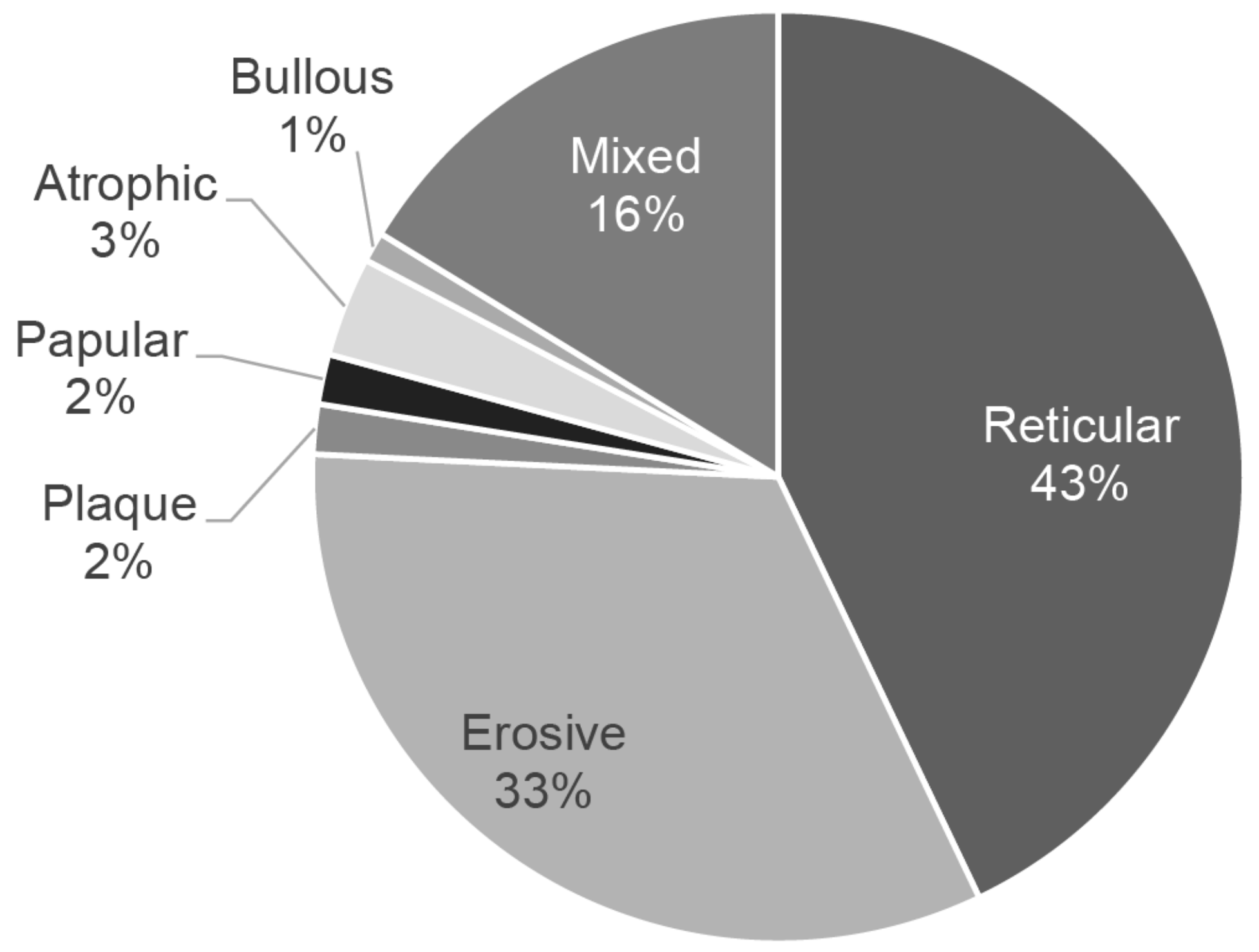

Figure 3

Clinical visual type classification of lesions 
- Gingivobuccal fold $\quad$ Buccal mucosa-Gingivobuccal fold

140 Lip $\square$ Palate $\square$ Tangue $\backsim$ Gingiva $\backsim$ Buccal mucosa

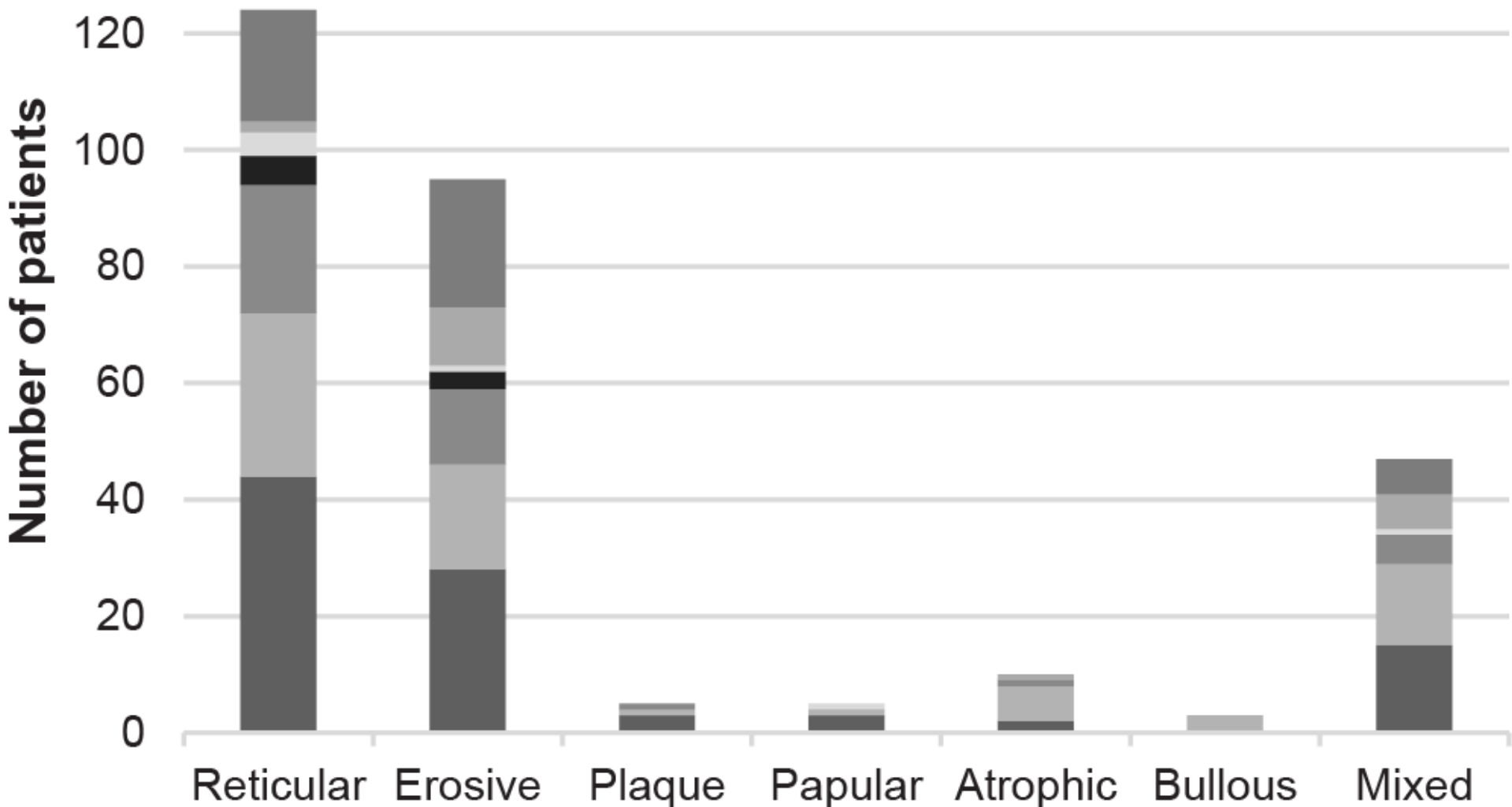

Figure 4

Number of lesions by site 


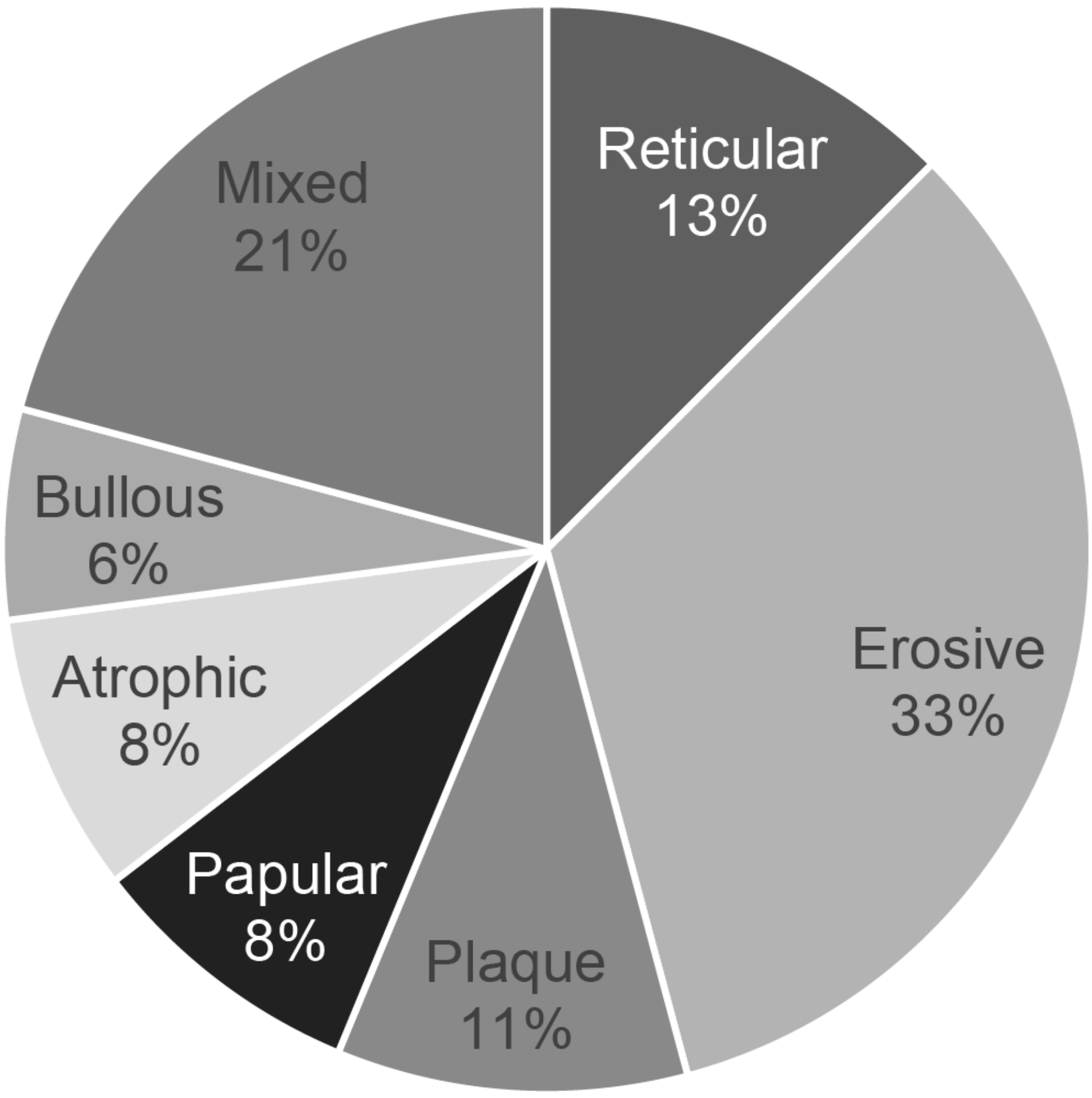

Figure 5

Clinical type of biopsy performed 

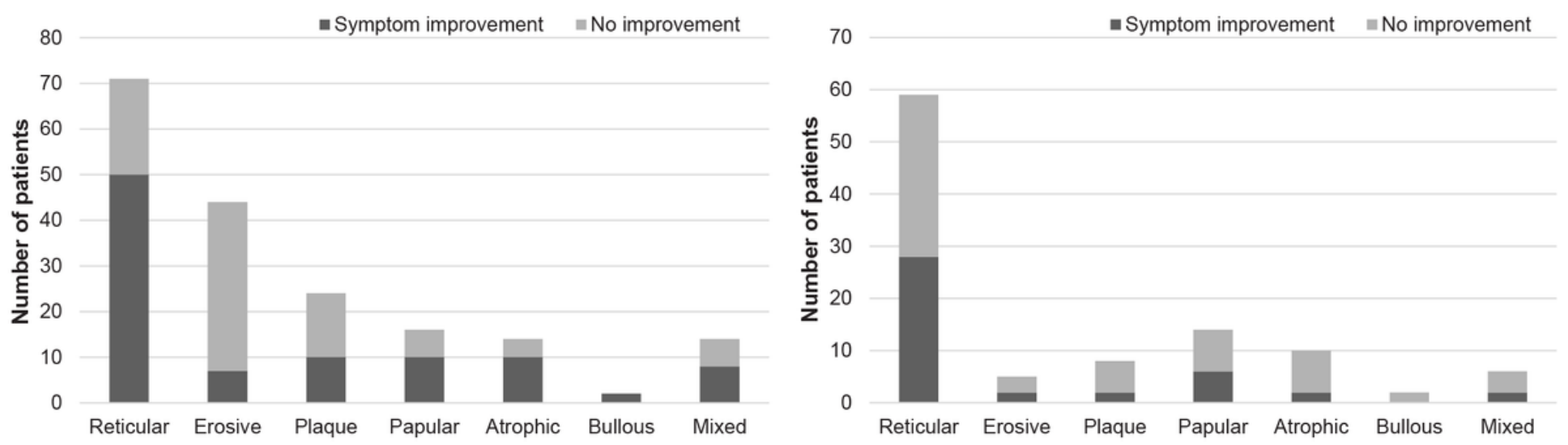

Figure 6

a) Percentage of symptom improvement in cases prescribed topical steroids. b) Symptom improvement rate in non-prescribed topical steroids 